\title{
Lack of conservation of bacterial type promoters in plastids of Streptophyta
}

\author{
Vassily A Lyubetsky*, Lev I Rubanov and Alexandr V Seliverstov
}

\begin{abstract}
: We demonstrate the scarcity of conserved bacterial-type promoters in plastids of Streptophyta and report widely conserved promoters only for genes $p s a A, p s b A, p s b B, p s b E$, rbcL. Among the reasonable explanations are: evolutionary changes of sigma subunit paralogs and phage-type RNA polymerases possibly entailing the loss of corresponding nuclear genes, de novo emergence of the promoters, their loss together with plastome genes; functional substitution of the promoter boxes by transcription activation factor binding sites.
\end{abstract}

Reviewers: This article was reviewed by Dr. Arcady Mushegian, and by Dr. Alexander Bolshoy and Dr. Yuri Wolf (both nominated by Dr. Purificación López-García).

\section{Background}

Genes evolve at different rates. Various hypotheses try to explain, or at least to correlate, the evolutionary rate (sequence conservation) and the functional properties of the protein-coding gene. As far as we know, there is no published evidence on searching for the plastid promoters at the genome scale. This problem should probably be addressed separately for nuclear, plastome and mitochondrial genomes, different taxonomic lineages and different RNA polymerase types. In particular, multisubunit RNA polymerase (PEP), which has the core enzyme encoded in plastome and the sigma subunit in nucleome, binds bacterial type promoters (PEP-promoters); and monosubunit RNA polymerases (NEP), which is nucleome-encoded, binds NEP-promoters. Here we report a study of PEPpromoters of plastome genes in representatives of the green line (Viridiplantae, including Chlorophyta and Streptophyta; Euglenozoa, Rhizaria, in particular Cercozoa; Glaucocystophyceae) and the red line (Rhodophyta, stramenopiles, including Bacillariophyta, Pelagophyceae, Raphidophyceae, Xanthophyceae; Cryptophyta, Haptophyceae, Apicomplexa). Add. file 1 describes the complete list of studied species with plastids, organized according to the NCBI Taxonomy. Plastid genes are

\footnotetext{
* Correspondence: lyubetsk@iitp.ru

1 Institute for Information Transmission Problems of the Russian Academy of Sciences, 19, Bolshoy Karetny per., Moscow, 127994, Russia
}

Full list of author information is available at the end of the article believed to be evolutionarily conserved across large taxonomic lineages [[1], section 9.7c], although the authors are unaware of systematic studies on their promoters conservation. Instead, there is ample published research on the promoter comparisons within small lineages, largely the studies of the promoters and their transcription factors in gamma- and alpha-proteobacteria [2]. Further, some pairs of closely related species have been shown to possess largely diverged promoters $[3,4]$. We have reported an evolutionary labile promoter for the $n d h F$ gene in a narrow lineage of dicotyledonous angiosperm plants and described four different promoter types, which are likely to have replaced each other during evolution [5].

In this study we aimed at searching for widely conserved PEP-promoters in plastomes of the above mentioned taxa. By "widely conserved" we mean the cases when the regions upstream of orthologous genes across the high-level taxonomic divisions can be aligned. The promoters confined to only vascular plants or the red line lineages are not examined here (e.g., the NEP-promoter of gene $c l p P$ in vascular plants). In our analyses using the fixed consensus as a query produced massive under-predictions, or, alternatively, massive over-predictions, which suggests that querying without taking into account the alignment of 5 -leader regions is obviously misleading. 


\section{Materials and methods}

The regions of up to $1000 \mathrm{bp}$ length upstream from all protein-coding genes (90 genes per species at average) in plastomes of species listed in Additional file 1 were extracted from GenBank, and multiple alignments of the regions were constructed. Searches of promoters were conducted using two original algorithms: the first to preselect leader regions with candidate PEP-promoters (several candidates were found per region), and the second to build a multiple alignment keeping one of the candidate promoters in each of the regions. The alignment was constructed to reveal the two bacterial type boxes and cover the taxonomic diversity of the above mentioned lineages as wide as possible. In a positive prediction, the alignment of the boxes, linker and some flanking regions was required to have a good quality (see below). Otherwise, a negative prediction is produced and a PEP-promoter is not detected with our method. Evidently "positive prediction" means the prediction of a PEP-promoter and "negative prediction" means the lack of positive prediction. Notably, the positive predictions contained experimentally proved PEP-promoters and often their TG-extensions, which indicates that these are not false positives. Also, in all negative predictions the alignment had a considerably lower quality compared to the minimal quality among all positive predictions. All predicted PEP-promoters were located within approximately $40 \mathrm{bp}$-long highly conserved regions flanked by less conserved 3'areas and highly variable 5 '-areas.

The idea of the first algorithm. Given is a set of $n$ leader regions. The goal is to find a subset of the set with one potential promoter in each region such that their total pair-wise similarity is maximal comparing to any other collection of potential promoters in that subset; the subset size is simultaneously maximized. In order to increase search speed, randomly selected regions are set as "linked" and the promoter similarity is estimated only within the linked pairs of regions. It formally means that we consider a graph with $n$ vertices, each assigned a leader region, but only linked regions are connected by an edge in the graph. As a result, the complexity of comparing all pairs of candidate promoters to determine their total similarity is reduced in our algorithm by means of considering a large number of randomly defined sets of edges, i.e. randomly constructed graphs with $n$ vertices assigned the same regions but connected by different edges. By doing so, the computing time becomes square to number $n$ of the regions and cubic to their average length. The algorithm is designed for effective parallelization to enable mass processing of large amounts of long regions in feasible time. The enhanced performance of the parallel implementation allows to compute a solution closer to the maximum quality of the alignment. The algorithm is highly scalable and provides for the approxi- mately linear growth of performance with the number of available processors up to 2000 .

The idea of the second algorithm. Along a fixed phylogenetic species tree, the algorithm aligns leader regions with respect to one of the candidate promoters selected by the first algorithm, from the promoter start up to the start codon. It uses a common observation that promoters, as well as transcribed regions, can be well aligned, in contrast to the region upstream of the promoter. The algorithm takes a non-binary (which is often the case) species tree and during the run reduces it to a binary tree in a variety (or even all) possible ways. Each leaf of the tree bears an orthologous gene leader region from the corresponding species. The alignment is constructed as follows. First, each leaf is assigned a nucleotide frequency distribution at each position of the sequence: the distribution contains a unity for the observed nucleotide type and three zeros for the unobserved. A zero distribution contains four zeros. Then, at each inner node, two distribution sequences at its descendant nodes are aligned by any applicable algorithm, with an award for matching two distributions not pre-defined, but calculated anew at each position $j$ taking into account the length of each descendant branch. The award is estimated as a scalar square of the difference between two nonzero distributions weighted for different nucleotide types. The penalty for inserting a gap symbol (i.e., for the alignment of zero and nonzero distributions) is a decreasing function of the number of contiguous gaps: the longer the gap region, the lower the penalty. Two zero distributions are forbidden to align. At each position of the alignment, the distribution in the ancestral sequence is a half-sum of the two distributions in the descendants. When the root distribution sequence is constructed, the algorithm projects the gaps along the tree to its leaves onto the extant sequences, thus obtaining the final multiple alignment. The complexity is linear to the number of leaves. Different binary tree resolutions are compared on the basis of the corresponding alignment quality, which is estimated as follows: $\left(N_{a}+N_{s}\right) b+\sum_{j=1}^{N_{s}}(b+s)\left(l_{i}-1\right)+N_{b} c$, where $N_{a}$ is the number of totally conserved (containing the same character) single columns, $N_{s}$ - the number of totally conserved regions (two or more contiguous totally conserved columns, $l_{i}$ is the number of columns), $N_{b}$ - the number of "nearly" conserved columns (with one non-matching character); $b, c$ and $s$ are parameters. Computing an alignment of 16 sequences with the length of 120-223 bases requires less than one second on a $3 \mathrm{GHz}$ Pentium-4 PC. The automatically computed alignments were manually checked and minor corrections were introduced if so required. Both algorithms are implemented as 32-bit command line utilities written in ANSI C, which can be 
compiled with many popular compilers and run under Windows or Linux. The algorithms and their detailed descriptions are available from [6,7].

Testing of the algorithms and their comparison with "common" local alignment algorithms (see the introduction and the list of references in [8]) are described in [9$11]$.

\section{Results}

Table 1 contains the species from add. file 1 predicted to possess at least one widely conserved promoter in the plastome. Predictions are identical for their close relatives with a corresponding orthologous gene (not shown). Within flowering plants the promoter sequences are similar and well aligned, therefore we illustrate results on Arabidopsis thaliana and Spinacia oleracea only. The five positive predictions are described below. Our analyses suggest that widely conserved promoters are absent elsewhere in streptophyte plastomes.

Gene $\boldsymbol{p} \boldsymbol{s} \boldsymbol{b} \boldsymbol{A}$ (protein D1 of the photosystem II active center) in plastomes. Promoters of this chloroplast gene were experimentally studied in selected species, including Arabidopsis, mustard, and spinach [3,12,13], for which our predictions are in good agreement with the experiment. The algorithm predicted candidate conserved promoters upstream of this gene in most Streptophyta, primary and secondary endosymbionts, Bigelowiella natans from the Chlorarachniophyceae, and Cyanophora paradoxa from the Glaucocystophyceae (ref. to Fig. 1, psbA). The gene alignments are given in Fig. 1, per-site nucleotide frequency distributions are given in Fig. 2 (constructed with the Weblogo program [14]). We suggest that this ancient promoter with the consensus TTGACA-15-TGTwATAmT is ancestral for at least all Streptophyta. The linker between the boxes is usually 18 bases long, but is 17 bases in Cycas taitungensis, Adiantum capillus-veneris, Staurastrum punctulatum, Mesostigma viride and B. natans. Many predictions possess the 5 -extension (TG or TGTG) of the "-10" box, which enhances the promoter efficiency. In the gymnosperm C. taitungensis, the predicted "-35" box essentially differs from the alignment consensus and the bacteriallike promoter. The $p s b A$ promoter was not found in the hornworts Anthoceros formosae, although in other bryophytes it is highly conserved. In the early emerging alga Chlorokybus atmophyticus only the "-35" box was identified, while the complete promoter was found in M. viride. Two dodder species (Cuscuta gronovii, C. obtusiflora) with a largely reduced plastome also lack the $p s b A$ promoter, which, however is found in their close relatives $(C$. exaltata, C. reflexa) and most angiosperm plants. The lack of promoters correlates with the reduction of genomes: Cuscuta gronovii and C. obtusiflora do not photosynthesize and lack most of the photosynthetic genes.
Although the $p s b A$ gene retains an open reading frame, it lacks the PEP-promoter and is probably poorly expressed compared to photosynthetic species.

Gene psbB (a chlorophyll apoprotein of photosystem II CP47) in plastomes of Streptophyta. For this gene, the transcription start is experimentally identified in spinach (S. oleracea) [15]; it adjoins the 3 '-end of the accordingly named sequence in Fig. 1, psbB. A conserved promoter is predicted in most vascular plants: in angiosperms $(A$. thaliana, S. oleracea), gymnosperms (Cycas taitungensis, Cryptomeria japonica, Welwitschia mirabilis, Pinus spp.) and pteridophytes (Adiantum capillus-veneris, Angiopteris evecta, Psilotum nudum, Huperzia lucidula). A related promoter is predicted in some algae (Chaetosphaeridium globosum, Chara vulgaris, Staurastrum punctulatum, Zygnema circumcarinatum, Chlorokybus atmophyticus, Mesostigma viride), ref. to Fig. 1, psbB. This promoter is highly conserved in C. taitungensis, $C$. japonica, pteridophytes and streptophyte algae C. globosum, C. vulgaris, S. punctulatum, and less conserved in $Z$. circumcarinatum, C. atmophyticus and $M$. viride. It possesses the "-10" box TG-extension. In the early branching C. atmophyticus and M. viride, several potential promoters are predicted in 5'-leader regions; however these cannot be unambiguously added to the alignment of Streptophytina (Fig. 1, psbB), especially in the regions between the boxes and start codons. Therefore, the promoters closest to the start codon are selected and shown for C. atmophyticus and M. viride. In bryophytes (Aneura mirabilis, Anthoceros formosae, Marchantia polymorpha, Physcomitrella patens), a conserved promoter was not found. Notably, the $p s b B$ sequence of $A$. mirabilis is annotated as a pseudogene in NCBI GenBank. The usual linker of $18 \mathrm{bp}$ between the boxes is reduced to $17 \mathrm{bp}$ in W. mirabilis and some algae (C. atmophyticus, S. punctulatum, Z. circumcarinatum). In the pines Pinus koraiensis and $P$. thunbergii, the sequence differences are not shown (they occur in between the end of the sequence in Fig. 1, $p s b B$ and the conserved processing site shown in Fig. 3).

Gene $\boldsymbol{p s} \boldsymbol{b} \boldsymbol{E}$ (photosystem II cytochrome b559 protein alpha subunit) in plastomes of Streptophyta. Promoters were predicted in most land plants and the algae Chaetosphaeridium globosum, Staurastrum punctulatum, Zygnema circumcarinatum, ref. to Fig. 1, psbE. Negative predictions were obtained for the algae Chara vulgaris, Chlorokybus atmophyticus and Mesostigma viride, even though the region is conserved in their closer relatives. This gene is a pseudogene in the Aneura mirabilis plastome.

Gene $r b c L$ (the large subunit of ribulose-1,5-bisphosphate carboxylase) in plastomes of Streptophyta. The promoter was experimentally characterized in spinach $(S$. oleracea) [13], and mustard (Sinapis alba) [12]. It was predicted in all land plants and in the streptophyte algae 
Table 1: Estimated coordinates of the transcription initiation sites of the predicted PEP-promoters

\begin{tabular}{|c|c|c|c|c|c|}
\hline Species & psaA & psbA & $p s b B$ & psbE & $r b c L$ \\
\hline $\begin{array}{l}\text { Arabidopsis } \\
\text { thaliana }\end{array}$ & Ex -188 & Ex -77 & -170 & -125 & -177 \\
\hline Spinacia oleracea & Ex -179 & Ex -82 & -175 & -150 & -176 \\
\hline Cycas taitungensis & Ex -156 & -60 & Ex -170 & -141 & -156 \\
\hline $\begin{array}{l}\text { Cryptomeria } \\
\text { japonica }\end{array}$ & Ex -142 & -58 & Ex -142 & -137 & -161 \\
\hline Pinus koraiensis & Ex -158 & -52 & -193 & -148 & -136 \\
\hline Pinus thunbergii & Ex -158 & -52 & -180 & -145 & -127 \\
\hline $\begin{array}{l}\text { Welwitschia } \\
\text { mirabilis }\end{array}$ & Ex -156 & Ex -51 & -271 & -31 & -136 \\
\hline $\begin{array}{l}\text { Adiantum capillus- } \\
\text { veneris }\end{array}$ & Ex -163 & Ex -55 & Ex -291 & -191 & -157 \\
\hline Angiopteris evecta & Ex -152 & Ex -69 & Ex -181 & -142 & -148 \\
\hline Psilotum nudum & Ex -147 & Ex -53 & Ex -178 & -127 & -140 \\
\hline Huperzia lucidula & Ex -153 & Ex -55 & Ex -187 & -134 & -150 \\
\hline $\begin{array}{l}\text { Anthoceros } \\
\text { formosae }\end{array}$ & Ex -155 & $=$ & $=$ & -143 & -160 \\
\hline Aneura mirabilis & Pseudo & Ex -54 & Pseudo & Pseudo & -148 \\
\hline $\begin{array}{l}\text { Marchantia } \\
\text { polymorpha }\end{array}$ & Ex -149 & Ex -53 & $=$ & -132 & -124 \\
\hline $\begin{array}{l}\text { Physcomitrella } \\
\text { patens }\end{array}$ & Ex -161 & Ex -53 & $=$ & -145 & -143 \\
\hline Chara vulgaris & Ex -199 & -121 & Ex -179 & $=$ & -154 \\
\hline $\begin{array}{l}\text { Chaetosphaeridiu } \\
\text { m globosum }\end{array}$ & Ex -154 & Ex -57 & Ex -161 & -119 & -102 \\
\hline $\begin{array}{l}\text { Staurastrum } \\
\text { punctulatum }\end{array}$ & Ex -235 & Ex -59 & -190 & -154 & -219 \\
\hline $\begin{array}{l}\text { Zygnema } \\
\text { circumcarinatum }\end{array}$ & Ex -157 & Ex -58 & -159 & -122 & -168 \\
\hline $\begin{array}{l}\text { Chlorokybus } \\
\text { atmophyticus }\end{array}$ & $=$ & $=$ & -266 & $=$ & $=$ \\
\hline Mesostigma viride & $=$ & Ex -53 & -89 & $=$ & $=$ \\
\hline $\begin{array}{l}\text { Bigelowiella } \\
\text { natans }\end{array}$ & $=$ & -136 & $=$ & $=$ & $=$ \\
\hline $\begin{array}{l}\text { Cyanophora } \\
\text { paradoxa }\end{array}$ & $=$ & -61 & $=$ & $=$ & $=$ \\
\hline
\end{tabular}

Coordinates are relative to the start codon. The "Ex" means the presence of the 5'-extension TG of the "-10" box, "Pseudo" marks a negative prediction for the pseudogene, "=" - a negative prediction for the functioning gene.

Chaetosphaeridium globosum, Chara vulgaris, Staurastrum punctulatum, Zygnema circumcarinatum, ref. to Fig. 1, rbcL.

Gene psaA (apoprotein A1 of photosystem I P700) in plastomes of Streptophyta. Promoter and the transcription initiation site for this gene were experimentally characterized in Arabidopsis thaliana [16]. In Aneura mirabilis it is a pseudogene. The promoter was predicted in almost all land plants and streptophyte algae, except for Chlorokybus atmophyticus and Mesostigma viride, see
Fig. 1, psaA. This promoter differs from all other predictions and the bacterial $\sigma-70$ promoter. Its "-10" box consensus is CATAAT, which differs from the bacterial type at the first position. At the 5 -end of the box a conserved putative extension is found with the consensus TrTGT. The predicted "-35" box is even more divergent from its counterparts, despite being located within a long conserved region.

Although the alignments shown Fig. 1 are unambiguous within the lineages, neither can be extended onto the 


\begin{tabular}{|c|c|c|c|c|c|}
\hline & Magnoliophyta & Arabidopsis thaliana & TTGGTTGACATGGCT-ATATAAGTCATGTTATACTGTTTCATAACAA & -74 & c1444 \\
\hline & & Spinacia oleracea & TTGGTTGACACGGG-CATATAAGGCATGTTATACTGTTGAATAĀCAA & -79 & c1278 \\
\hline & Cycadophyta & Cycas taitungensis & TCGATTCACGATA--TATATAAGTCATACTATACTGTTAAATAACAA & -57 & c1062 \\
\hline & Coniferophyta & Cryptomeria japonica & TTGGTTGACATACA-GATATGTCTCATATTATACTGTTGAATAACAA & -55 & c41765 \\
\hline & & Pinus koraiensis & TTGGTTGACATTGAT-ACATGGATCATATTATACTGTAAAATAACAA & -49 & c976 \\
\hline & & Pinus thunbergii & TTGGTTGACATTGAT-ACATGGATCATATTATACTGTAAAATAACAA & -49 & c976 \\
\hline & Gnetophyta & Welwitschia mirabilis & ATAGTTGACTTTAAT-AAACCATTTCTGTTATACTGTTAAAATAACA & -48 & c899 \\
\hline & Moniliformopses & Adiantum capillus-veneris & TTGGTTGACACGGAT-AGGTTTTT-GTGATATGCTACATAGTAACAG & -52 & 96368 \\
\hline & & Angiopteris evecta & TAAGTTGACATCAAT-AGATAAGTTGTGTTATACTATGAAGTAACAA & -66 & c8986 \\
\hline & & Psilotum nudum & TAAGTTGACATATAT-GGAAAGATCATGTTATACTTCAAATCAACAG & -50 & c8476 \\
\hline $\operatorname{cs} b A$ & Lycopodiophyta & Huperzia lucidula & TGGGTTGACACAAA-AAGAAAGATTGTGTAATATTATGGAATAACAA & -52 & c67506 \\
\hline & Marchantiophyta & Aneura mirabilis & GATGTTGACATAC-TAATGGGATATGTGTAATAATATGGGTTAACAG & -51 & 27556 \\
\hline & & Marchantia polymorpha & TTAGTTGACATAA-TCATATGTTATGTGTAATACTATAAGTTAACAA & -50 & 28368 \\
\hline & Bryophyta & Physcomitrella patens & TCAGTTGACATAA-TAATACATTTTGTGTAATACTATAAATTAACAA & -50 & c54280 \\
\hline & Charophyceae & Chara vulgaris & CTAGTTGACATTT-TTATACTTTACATACTATAATATCTAATAACAA & -118 & 41097 \\
\hline & Coleochaetophyceae & Chaetosphaeridium globosum & TAGGTTGACATTAGTTATACGT-TTGTGCAATACTAAATATTAACAA & -54 & c66153 \\
\hline & Zygnemophyceae & Staurastrum punctulatum & AAGGTTGACAGCT-TAAGGTTAAT-ATGTAATAATATAATTTAACAA & -56 & 65382 \\
\hline & & Zygnema circumcarinatum & TTAGTTGACAACAG-CATTAACTATCTGTAATAATATAAATTAACAA & -55 & 52018 \\
\hline & Mesostigmatophyceae & Mesostigma viride & TTATTTGACAAATA-AACATCATTT-TGGCATAATAATAATCAACAA & -50 & c4629 \\
\hline & Chlorarachniophyceae & Bigelowiella natans & TTTTTTGATTAATATAA-ATTAATTA-GTTATAATATTATAGAGTAA & -133 & c39582 \\
\hline & Glaucocystophyceae & Cyanophora paradoxa & AAGCTTGACAAAT-TAGACCATTAA-TATTATTATAAGATTTAACGA & -58 & 89183 \\
\hline & Magnoliophyta & Arabidopsis thaliana & CCCATTGCATATTGGTACTTATCGGATATAGAATAGATCCG & -171 & 72371 \\
\hline & 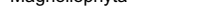 & Spinacia oleracea & CCCATTGCGTATTGCTACTTATCGAGTATAGAATAGATTTGT & -176 & 71047 \\
\hline & Cycadophyta & Cycas taitungensis & CACATTGTGCATTGGTACACATAAATGATAAAATATTTACG & -171 & 76344 \\
\hline & Coniferophyta & Cryptomeria japonica & CACATTGTATATTGATACATATAAATGATAAAATATATCCG & -143 & 4013 \\
\hline & & Pinus koraiensis & TACATTGTGTATTGGTACATACAAACGATAAAATATCTTTG & -194 & 51198 \\
\hline & & Pinus thunbergii & TACATTGTGTATTGGTACATACAAACGATAAAATATCTTTG & -181 & 52424 \\
\hline & Gnetophyta & Welwitschia mirabilis & TCACTTGGACCCAAGCCTCC-CTTTTTCTACTATATATAAT & -272 & 56136 \\
\hline & Moniliformopses & Adiantum capillus-veneris & TACGTTGTTACATGGGGAATGAAAATGCTAAAATATTCACG & -292 & 67792 \\
\hline$p s b$ & & Angiopteris evecta & CACATTGTTATGCAAAATCTGTGAATGCTAGAATATCTATG & -182 & 76067 \\
\hline & & Psilotum nudum & CACATTGTTGCACAAATTGTGCAAATGTTAAAATATCTCTG & -179 & 71406 \\
\hline & Lycopodiophyta & Huperzia lucidula & TCCATTGCGATGTTAAACGCATGGATGTTAAACTATTTCTG & -188 & c14368 \\
\hline & Charophyceae & Chara vulgaris & ATTCTTGGACGGTCAAGTTATAAAATGGTATAATATATAAA & -180 & 112833 \\
\hline & Coleochaetophyceae & Chaetosphaeridium globosum & AATATTGATATATAAGACAAATTAATGTTAAAATAATAATT & -162 & c35896 \\
\hline & Zygnemophyceae & Staurastrum punctulatum & TGTGTTGTTCTGAT-AGAAAAGAAATGATACAATCAAAATG & -191 & c103405 \\
\hline & & Zygnema circumcarinatum & TTAGTTGTAATCTC-ATAAGAGATAGAGTACAATGGAATTG & -160 & 7207 \\
\hline & Chlorokybophyceae & Chlorokybus atmophyticus & AGACTTGTTATCCTAATTAG-TTTGGTATATAGTTTGTTTT & -267 & 13435 \\
\hline & Mesostigmatophyceae & Mesostigma viride & TTAGTTGTTATAATTATACGTTAATAATTATAAATGTATTT & -90 & 7825 \\
\hline & Magnoliophyta & Arabidopsis thaliana & TGCGTTGCTGTGTCAGAAGAGGATAGCTATACTGATTCGGTAGAC & -120 & c64322 \\
\hline & & Spinacia oleracea & TGCCTTGCTGTGTCAGAAGAAGGATAGCTATACTGATTCGGTATAC & -145 & c63209 \\
\hline & Cycadophyta & Cycas taitungensis & TGTATTGCTGTGTCAGAGGAAGGCTAGCTATACCGGTCCAATATAC & -136 & c68353 \\
\hline & Coniferophyta & Cryptomeria japonica & TATATTGCTATGTTAGAAGCAGGCTAGCTATACTTAGTATACTTCA & -132 & 22819 \\
\hline & & Pinus koraiensis & TGTATTGCTGTGTCAGAAGAAAGCTAGCTATACTGGTCCAGTTATA & -143 & 35351 \\
\hline & & Pinus thunbergii & TGTATTGCTGTGTCAGAAGAAAGCTAGCTATACTGGTCCAGTAGAC & -140 & 35300 \\
\hline & Gnetophyta & Welwitschia mirabilis & TATATTGCTGTGTCATAAAAAAGTTGGTTATACTGGTCCAGTATTA & -26 & c49332 \\
\hline & Moniliformopses & Adiantum capillus-veneris & AACCTTGCCGCATTGTACGTGAAATAGCTATACTGACCCAGCATAT & -186 & c60502 \\
\hline & & Angiopteris evecta & TATCTTGCTGCGTCAAAAGAAGGCTAGCTATACTGTTCTAGTATAT & -137 & c69606 \\
\hline & & Psilotum nudum & TCTCTTGCTGTATAGGAAAAAAGATAGCTATACTGATACTATATAT & -122 & c64390 \\
\hline & Lycopodiophyta & Huperzia lucidula & TGTCTTGCTGCGTCAGAGGACACTAGCTATACTAGTCTAGTATAC & -129 & 24315 \\
\hline & Anthocerotophyta & Anthoceros formosae & TACCTTGCTTCGTTGAAAGAACGCTAGCTATACTTATTTAGTATGC & -138 & c82498 \\
\hline & Marchantiophyta & Marchantia polymorpha & TATCTTGCTGCGTAAAAAGAACATTAGCTATACTAAGTTAGTATGC & -127 & c63554 \\
\hline & Bryophyta & Physcomitrella patens & TGTCTTGCTACGCTAAAACAACCCTAGATATACTTATTTAGTATGC & -140 & 17391 \\
\hline & Coleochaetophyceae & Chaetosphaeridium globosum & TCTCTTGCTGGCTGGTTAGTTAAATAGGTATACTATAATTGTACGT & -114 & c58320 \\
\hline & Zygnemophyceae & Staurastrum punctulatum & GGCCTTGCTGTCTTAAAGAAATCTTAGTTATACTTACTTAGCATGT & -149 & 61021 \\
\hline & & Zygnema circumcarinatum & AGTGTTGCTCTATAAAAACAATGTGAGGTATACTTAGTTAGCAGCT & -117 & c95644 \\
\hline & Magnoliophyta & Arabidopsis thaliana & TAGGTTGCGCTATACATATGAAAGGAATATACAATAATGATGTATTT & -172 & 54958 \\
\hline & & Spinacia oleracea & TGGGTTGCGCCATATATATGAAAGAGTATACAATAATGATGTATTT & -171 & 53825 \\
\hline & Cycadophyta & Cycas taitungensis & AGGGTTGCGCCATACATAAAGAACATTATACAATAATAGTGTATTT & -151 & 59064 \\
\hline & Coniferophyta & Cryptomeria japonica & TGGGTTGCGTCATACATACATAACATGATACAATATCACTTGAAAG & -157 & c30177 \\
\hline & & Pinus koraiensis & TGGGTTGCGTCATACATAAAGAACATTATACAATGAGAGTGTATCT & -131 & c44225 \\
\hline & & Pinus thunbergii & TGGGTTGCGTCATACATAAAGAACACTATACAATGAGAGTGTATCT & -122 & c44473 \\
\hline & Gnetophyta & Welwitschia mirabilis & TGGGTTGCATTATATGGAAAAAACAATCTAAAATGATAGTGTATT'T & -131 & 42893 \\
\hline & Moniliformopses & Adiantum capillus-veneris & TTAGTTGCACCCCGCATCGGACGCGGTATAAAATAATAATGTTCCA & -152 & 51894 \\
\hline & & Angiopteris evecta & TGGGTTGCATTATACAGAAAATAATTTATAGAATACTAGTGTCTCA & -143 & 60605 \\
\hline rbcL & & Psilotum nudum & TGGGTTGCATCATATAGCAACTGCAATATAAAATAATAGTGTTTCC & -135 & 55824 \\
\hline & Lycopodiophyta & Huperzia lucidula & TGGGTTGCATCACGTATCAAAAGCAATATACAATGATAATGTTTTA & -145 & c33938 \\
\hline & Anthocerotophyta & Anthoceros formosae & TAGGTTGCATCATATACTAGAAATAATATACAATAGTAATGTTTTA & -160 & 72912 \\
\hline & Marchantiophyta & Aneura mirabilis & TGGGTTGCATTACGTCGGATAAGCAATATACAATAATGATGTTTCA & -143 & 52514 \\
\hline & & Marchantia polymorpha & TAGGTTGCATTACATATAAAAAACAATATACAATAATAATGTTTTA & -119 & 56355 \\
\hline & Bryophyta & Physcomitrella patens & TGAGTTGCATCAAATGTAGAAAATAATATACAATAATACTGTTTTG & -138 & c25866 \\
\hline & Charophyceae & Chara vulgaris & TGGCTTGTGTAGAGTAAATATTTATATATATAATATACGTACCGCC & -97 & 75969 \\
\hline & Coleochaetophyceae & Chaetosphaeridium globosum & TTAGTTGCGTCATCTATTCAAGAATGTGTATAATACAATATAGAAA & -149 & 50115 \\
\hline & Zygnemophyceae & Staurastrum punctulatum & TTAGTTGTTTTAATCAATGTATGTAGT-TACAATAAATTTGTAATA & -214 & 41614 \\
\hline & & Zygnema circumcarinatum & AGGGTTGCAGATGATAAAAAA-GTAATATATAATGAAGTTGCTGCT & -163 & c13185 \\
\hline & Magnoliophyta & Arabidopsis thaliana & TCCGTTGAGCACCCT-ATGGATATGTCATAATAGATCCG-AACACTTGC & -179 & \\
\hline & & Spinacia oleracea & TCCGTTGAGCGCCAC-ACGTCTATGTCATAATAGATCCG-AACACTTGC & -171 & c40552 \\
\hline & Cycadophyta & Cycas taitungensis & TCCATTGAGCACCTC-AGGGATATGTCATAATAAATTTG-AACACCTGC & -147 & c43428 \\
\hline & Coniferophyta & Cryptomeria japonica & TCCATTAAGCACCTA-TCAGATATGTCATAATAAATATGAACACCTGTC & -133 & 52692 \\
\hline & & Pinus koraiensis & TCCATTGAGCACCTC-GAAGATATGTCATAATAAAACTG-AACACCTGC & -149 & 72325 \\
\hline & & Pinus thunbergii & TCCATTGAGCACCTCAAAAGATATGTCATAATAGAATTG-AACACCTGC & -149 & 73819 \\
\hline & Gnetophyta & Welwitschia mirabilis & TCCATTGAGCGCCTCTTGTATTATGTCATAATAAAAAGGGAACACCTGC & -146 & c14264 \\
\hline & Moniliformopses & Adiantum capillus-veneris & TCCATCAGGCGCCGCT-AAGCCGTGTAATAATACCACCG-AAAGCCTAT & -154 & c40402 \\
\hline & & Angiopteris evecta & TCCATTAAGCACTTTT-TGATTGTGTAATAATAAAATTG-AATGCCTGC & -143 & c49417 \\
\hline psaA & & Psilotum nudum & TCCATTAAGCACTTC-GATATTGTGTAATAATAAGTTTT-AATACCTGC & -138 & c44788 \\
\hline & Lycopodiophyta & Huperzia lucidula & TCCATTAAGCACCTTT-GATATGTGTAACAATAATTTTG-AATACCTGC & -144 & 46994 \\
\hline & Anthocerotophyta & Anthoceros formosae & TCCATTAAGCACCTTT-GAGATGTGTCATAATAAAAATG-AATACTTGC & -146 & c59162 \\
\hline & Marchantiophyta & Marchantia polymorpha & TCCATTAAGCACCTT-AAAATTGTGTCATAATAAATTTG-AAGACCTGC & -140 & c47207 \\
\hline & Bryophyta & Physcomitrella patens & TCCATTAAGCACCTT-AAAGATGTGTCATAATAAATTTG-AATACCTGC & -152 & 35758 \\
\hline & Charophyceae & Chara vulgaris & TCCATTAAGCGCTCT-ATATATATGCCATACTACAGGTATGAAA-GTCT & -190 & 51107 \\
\hline & Coleochaetophyceae & Chaetosphaeridium globosum & TCCATCAAGCAC-CTAAAAAATGTGTCATAATTTATTAG-AACACTTAC & -145 & 69849 \\
\hline & Zygnemophyceae & Staurastrum punctulatum & TCCCTTTAGCACT-AAAAAAATATGCCATAATATAAATA-GAAACCTAC & -226 & c127624 \\
\hline & & Zygnema circumcarinatum & TCCATCAAACACTGT-GTGTGTGTGTCATAATACATTTTAGA-ACCTGC & -148 & c139440 \\
\hline
\end{tabular}

Figure 1 Predicted promoters upstream of genes psbA, psbB, psbE, rbcL, psaA. In the cells of first column only first occurrences of each taxon name are given. In yellow are the promoter boxes and the 5'-extension of the "-10" box. Numbers are the distance to the start codon; its location is given in the last column, prepended with "c" for complement sequences. In violet are the experimentally identified transcription initiation sites in Arabidopsis thaliana and Spinacia oleracea upstream of psbA, psbB, rbcL, psaA. 

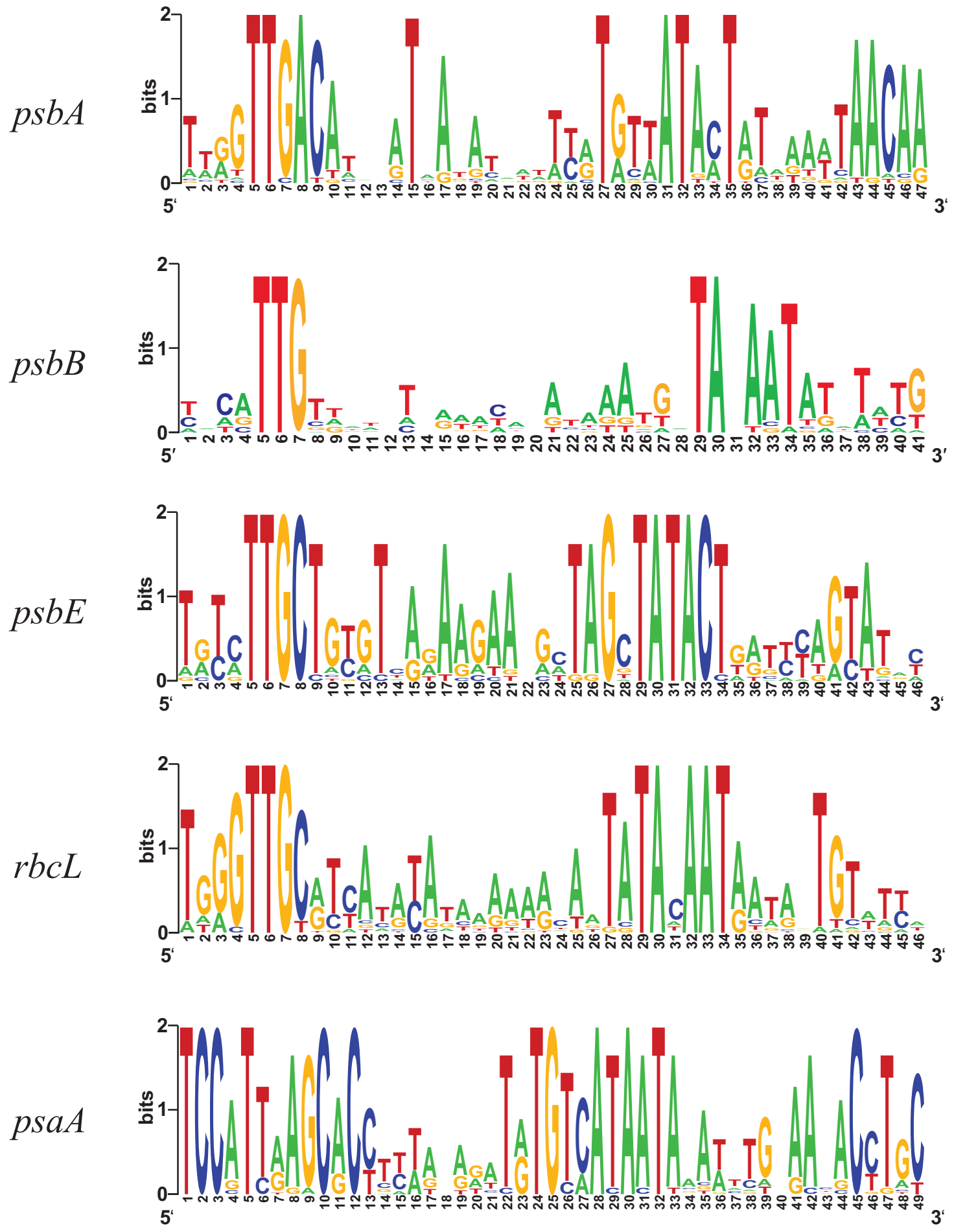

Figure 2 Nucleotide frequency distribution for the alignments shown in Fig. 1. 


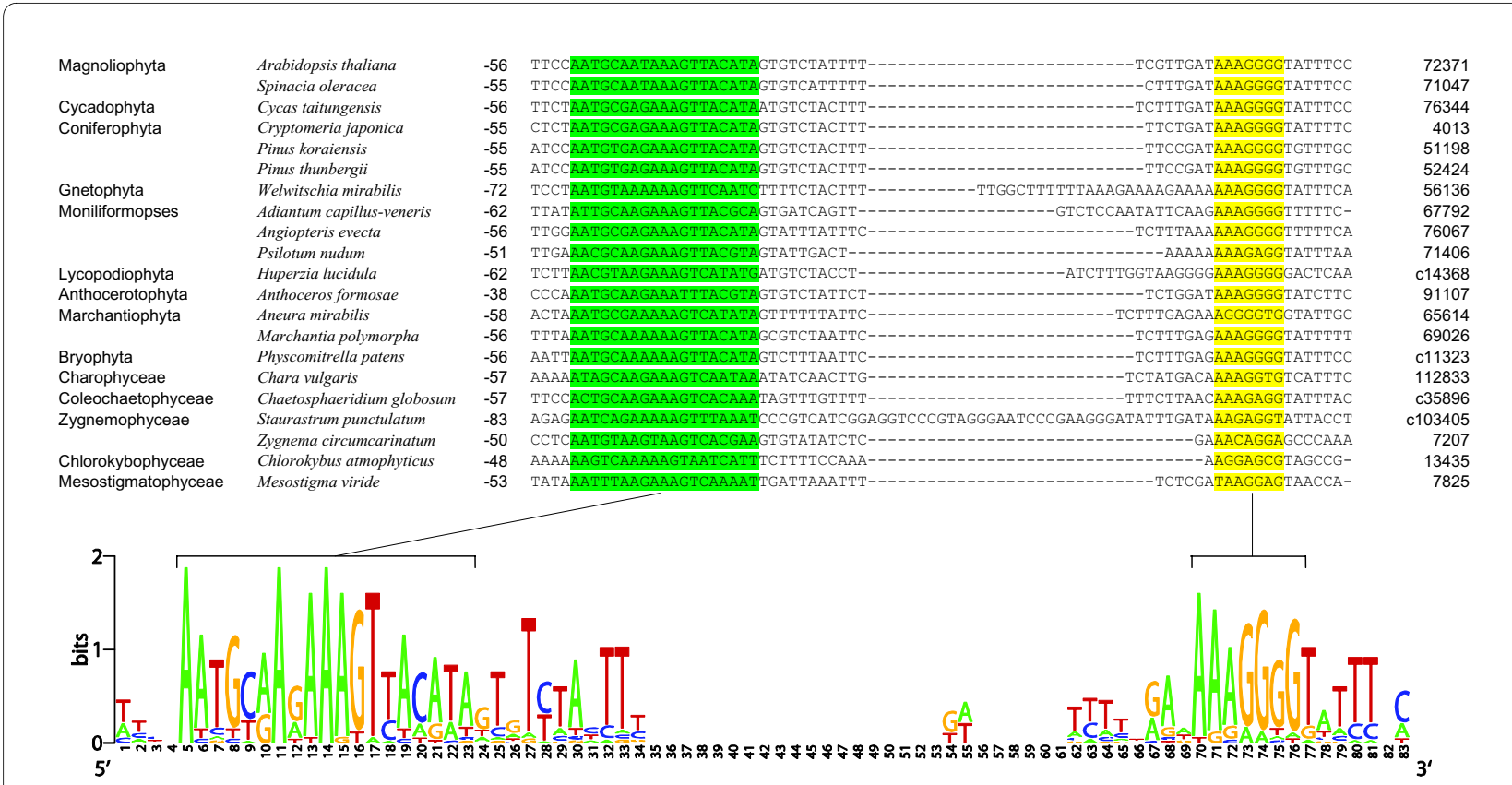

Figure 3 The 5 '-leader regions upstream of gene psbB. In the cells of first column only first occurrences of each taxon name are given. Numbers to the left of the sequences are distances from the 5'-edge to the start codon, which location is specified in the last column ("c" stands for complement sequences). In spinach the region is located precisely between the mRNA cleavage site and the start codon. Conserved putative mRNA-protein binding sites downstream of the cleavage site are shown in green. Conserved putative ribosome binding sites close to the start codon are in yellow.

Euglenozoa, Chlorophyta, Rhodophyta, Cryptophyta, diatom and other algae with plastids similar to those of the Rhodophyta, see add. file 1.

Normally, the entire promoter region, not only the boxes, is more conserved comparing to the rest of the leader region, which hampers distinguishing between regulated and non-regulated promoters.

We illustrate the comparison between wide and local conservations on the PEP-promoters of genes ycf1, rps4 and $p s a J$. The promoters were experimentally identified in Arabidopsis thaliana. These genes are among the 85 protein-coding genes in the plastome of $A$. thaliana. They are not widely conserved.

The $y c f 1$ gene encodes an unknown function protein and has PEP-promoter $y c f 1$-34 with a smaller distance between the "-35" and "-10" boxes than normally [3]. This promoter overlaps with NEP-promoter $y c f 1$-39. PEP-promoters very similar to $y c f 1-34$ with unambiguous multiple alignments of the 5'-UTR regions are found in most eudicotyledonous, magnoliid and basal magnoliophyte plants. Some species (including Cucumis sativus) possess a much longer 5'-UTR region, while in others (including Ranunculus macranthus) the $y c f 1$ PEP-promoter is not found. In monocotyledonous (Liliopsida), gymnosperm and pteridophyte plants possessing the $y c f 1$ gene, its putative PEP-promoters are found but differ considerably from those in eudicotyledons, magnoliids and the basal
Magnoliophyta. The promoter in A. thaliana is most similar to that from the cycadophyte Cycas taitungensis.

In $A$. thaliana the gene rps4 encoding ribosomal protein S4 has PEP-promotor rps4-123 [3]. Similar promoters with unambiguous 5'-UTR multiple alignment are found only in selected species of Brassicaceae: Arabis hirsuta, Barbarea verna, Crucihimalaya wallichii, Draba nemorosa, Lepidium virginicum, Lobularia maritima, Nasturtium officinale and Olimarabidopsis pumila. The plastomes of $B$. verna, D. nemorosa, L. maritima and $O$. pumila contain single nucleotide insertions in between the boxes;Arabis hirsute has a single nucleotide deletion. The promoter region is variable even across close species (Aethionema cordifolium, A. grandiflorum, Carica papaya, Citrus sinensis) but their 5'-UTR regions can still be well aligned.

A. thaliana was experimentally found to possess a Sig2dependent promoter upstream of gene psaJ encoding photosystem I active center subunit IX, with a 37 nucleotide-long 5'-UTR [17]. Although well aligned across all eurosids II, its 5'-UTR regions are conserved only within Brassicaceae and diverge already in C. papaya.

\section{Discussion}

Conserved promoters are found in the monophyletic Streptophyta and in two distant species, B. natans and $C$. paradoxa. Notably, even though $B$. natans belongs to the 
Cercozoa, its plastome is similar to that of green algae [18]. On the contrary, the plastome of C. paradoxa is different in many respects $[19,20]$.

There are many reasons why PEP-promoters upstream of the protein-coding plastome genes are scarce. Their loss may be related to the evolutionary changes of sigma subunit paralogs and phage-type RNA polymerases that lead to rapid replacements of the PEP-promoter. Indeed, the PEP sigma subunits vary already between maize, poplar and thale cress: e.g., maize possesses two Sig2 paralogs and lacks Sig4, while in poplar sig4 is a pseudogene, and thale cress possesses a Sig4 and only one Sig2, [21]. Also, promoters can be lost with their nuclear sigma subunitencoding genes, such as the Sig4-dependent $n d h F$ promoter in poplar [5]. Some dicotyledonous plants, including Arabidopsis and Nicotiana, have gained the additional phage-type RNA polymerase RpoTmp, which is active in chloroplasts and mitochondria of these plants but is missing from monocotyledonous plants (unpublished dissertation by K. Kühn, 2006). Only one phage-type RNA polymerase, RpoTp, is known from plastids of monocots (Zea, Triticum), two phage-type RNA polymerases - from plastids of dicots (Arabidopsis, Nicotiana): RpoTp in chloroplasts and RpoTmp in both chloroplasts and mitochondria. The moss Physcomitrella patens also has two phage-type polymerases, RpoT1 and RpoT2, which target both chloroplasts and mitochondria [22]. Promoters can emerge de novo, as has been shown, e.g., for the $n d h F$ promoter [5]. Others are lost together with plastome genes, e.g., the chlL promoter in flowering and some other plants (according to the GenBank records). Another possible factor in rapid promoter turnover in plastids may be tissue-specific differentiation of plastid types, especially in vascular and, particularly, flowering plants, which evolved a rich diversity of sigma subunits [21] and phage type RNA polymerases. Often the promoter boxes are functionally substituted by the transcription activation factor binding sites [4].

In parasitic, non-photosynthesizing plants, such as dicotyledonous dodder (Cuscuta spp.) and liverwort Aneura mirabilis, many chloroplast genes are pseudogenes [23] and promoters of these genes are lost too. The promoter conservation might become lower in the presence of alternative promoters. The promoter might have undergone rapid evolution [3,5] and become unrecognizable. It also might be located beyond the $1000 \mathrm{bp}$ distance from the start codon and thus be overlooked in our analyses.

Given these multiple reasons to expect fast evolution and rapid turnover of the chloroplast promoters, one may ask why some of them, such as the five promoters described above, are so widely conserved? One possible explanation is that three of the conserved promoters regulate the expression of the photosystem components and that the stability of the promoter structure is important to maintain high expression of genes $p s b A, p s b B, p s a A$; due to the light-dependent translation regulation of $p s b A$, a high amount of mRNA is built up in the dark and translated under light [24]. Conserved promoters upstream of $p s b A$ and $p s a A$ may also be required to form polycistronic mRNAs, which encode, along with the photosystem components, tRNA and proteins involved in translation that also have to be expressed at high levels: $p s b A$ appears to belong to the same operon as histidine tRNA, while $p s a A B$ and rps14 are in an operon with methionine tRNA. The psbEFLJ operon and $p s b B T H$ pet $B D$ operon might be formed likewise. The other conserved promoter regulates $r b c L$, the large subunit of a key enzyme involved in the carbon dioxide fixation during the Calvin cycle, the most abundant enzyme in the biosphere, whose gene also must be highly expressed. When a gene is highly transcribed and regulated by a single promoter, the selection pressure prevents any considerable change in the promoter's structure to provide for its effective binding to the polymerase.

Relatively lower conservation of the PEP-promoters of housekeeping genes (viz., tRNA, rRNA, ribosomal protein and PEP subunit-encoding genes, etc.) might be explained by the presence of NEP transcription: e.g., the rроB transcription is entirely NEP-mediated, although most genes possess both PEP and NEP-promoters. This is the case of the $y c f 1$ and $c l p P$ genes, which were experimentally shown in Arabidopsis thaliana to be under several promoters recognized by PEP with different subunits and two NEP, RpoTp and RpoTmp, [22].

Operonic organization and RNA polymerase competition are important factors explaining the effect of genome rearrangements on the evolution of promoters. Thus, the loss of the common $n d h F$ promoter and the emergence of a new one upstream of gene $n d h F$ in poplar (Populus alba, $P$. trichocarpa) concur with the deletion of a neighboring gene [5].

Some conserved promoters might be overlooked. For instance, the well studied $p s b C$ promoter is located within a coding region of other gene (according to the GenBank records) and its conservation cannot be assessed without estimating the synonymous vs. non-synonymous substitutions ratio, which is yet to be incorporated in our approach. Similar promoter-like regions were observed within other coding areas (unpublished data), but their role awaits explanation.

\section{Reviewers' comments \\ Reviewer's report 1}

Arcady Mushegian, Stowers Institute

The manuscript by Lyubetsky et al. examines the conservation of promoters in the choroplast genes of Streptophyta. The evidence is presented that, across large 
evolutionary distances (i.e., larger than the flowering plants clade) only a handful of promoter sequences contains conserved regions. This is an interesting observation suitable for publication in the Discovery Notes section of Biology Direct.

1) 1st paragraph: the authors assert that there is no published evidence on searching for promoters at the genome scale. This is not true and needs to be qualified: there are many papers about eukaryotes and several about either methods to detect or databases of detected promotors in various groups of bacteria, some of which have been obtained using intergenomic conservation as one of the criteria. Citing the research behind J.ColladoVides databases or RegulonDB might be in order.

Response: This sentence lacks the word "..plastid.." which occurs widely in our text and is present in the title. We now refer to the works by professor Collado-Vides [2], which contain references to databases on promoters and regulation factors including the RegulonDB database. These databases and other citations in [2] are related to selected gamma-, alpha-proteobacteria and eukaryotic nucleoms. We do not see them as directly related to the "searching for the plastid promoters at the genomic scale". Particularly, the RegulonDB database does not contain photosynthesis and many other plastome genes because they lack in $E$. coli. The intergenomic conservation ideology is used in our algorithms [6,7] but in a form different from that in [2].

2) Methods: references 4 and 5 are links to the authors' website with the documentation of their software. Why the reliance on the original code instead of the established methods of motif search and sequence alignment? Please explain crucial differences in the algorithms and how the homegrown ones were tested.

Response: Studies $[9,10]$ report testing of the "first" algorithm in our approach in the comparison with established local alignment algorithms. The "second" algorithm and its testing was reported during a conference [11]. Widely used "standard" programs did not produce better promoter predictions (they are described in [8] and many related references). An explanation might be that we define a PEP-promoter as two boxes separated by a region (sometimes with a TG extension) variable in terms of structure and length; the imposed requirements are the degree of the variability of this region, the linker between the "-10" box and the start codon and the 5 '-end of the "-35" box. The alignment of leader regions was built based on the precomputed two-boxed structures. It is more efficient to build it along a (usually known) species tree and not construct the alignment and the tree anew together as some approaches do. Ideologically the algorithms are described in the text, full details are given in $[6,7]$ and demonstrate their different performance comparing to other published methods.
3) A suggestion that may help to provide a more complete picture of the evolutionary trends in chloroplast promoter conservation: A. thaliana chloroplast has 85 protein-coding genes. Can we have a table that shows, for each gene, how broadly its promoter is conserved?

Response: The "Results" section now contains an analysis of PEP-promoter conservation upstream some coding genes in A. thaliana. An analysis of all 85 genes would be a subject for a separate publication. We show (as also noted in [5]) a typical problem in finding non-widely conserved promoters. Thus, well studied gene $n d h F$ in $A$. thaliana is found to have only one PEP-promoter out of the four types known in Magnoliophyta, which is conserved across the Brassicaceae and predicted in all sequenced eurosids II and in Vitis vinifera [5]. Chloroplast PEP-promoters are experimentally unidentified for many coding genes in A. thaliana, while for many they are [3]. These promoters are conserved also in the Brassicaceae but already in eurosids II their recognition depends on imposed cut-offs and requires biological validation. For widely conserved promoters over-prediction is much lower than for promoters conserved within a thin lineage where the leader regions did not diverge to a noticeable extent.

\section{Reviewer's report 2}

Alexander Bolshoy, University of Haifa (nominated by Purificación López-García, Université Paris-Sud)

In the paper of Lyubetsky et al. conservation and variability of the plastid promoters is studied, and, to the best of my knowledge, for the first time at the whole genome level. Undoubtedly, the problem is important and nontrivial. The authors obtained unexpected result: promoter regions in plastids are less conservative than corresponding coding sequences. To identify promoters the authors proposed an original method of searching short motifs surrounded by certain other motifs. Thus, the proposed article includes an interesting problem, original methods to solve it and non-trivial results of analysis of promoter regions. It makes this article suitable for publication in the Discovery Notes section of Biology Direct.

My remarks:

1) In Background section you use a term "lower conservation". Can you show how have you compared protein conservation with promoter conservation? Response: Comparing to the PEP-promoters, their regulated proteins are always widely conserved and well aligned. A family present in vascular pants is almost ubiquitous, while known widely conserved PEP-promoters are only five. PEP-promoters might be more abundant than NEPpromoters: the knockout of RpoTp-NEP is not lethal for A. thaliana, while the PEP-promoter loss (e.g. in Epifagus virginiana) entails the loss of numerous genes. The authors are unaware of detailed estimates. 
2) In Background section you use the term "widely" to indicate that the leader region sequences upstream orthologous genes can be aligned across high-level taxonomic divisions. Please, give some details for better understanding of the term "widely conserved"?

Please refer to Response \#3 to Yu.W.

3) In Background section the following phrase "... using the fixed consensus as a query produced massive underpredictions, or, alternatively, massive over-predictions..." needs some explanation.

Response: A simple approach to the promoter search is to define a conserved query mask. Using masks very close to, e.g., the bacterial sigma-70 consensus, will lead to under-predictions because reliable PEP-promoters of different structure will be overlooked. Using diverged masks will lead to numerous false predictions. We believe that using a fixed per-site nucleotide frequency queries is not a perspective.

4) Materials and methods. Please, give a short description of your algorithms.

Response: We developed an original approach to the promoters search. At the first stage we find a two-boxed signal via local multiple alignment (the first algorithm, ref. to Response to A.M \#2). For each leader region the algorithm predicts a number of candidate "-35" and "-10" boxes. The second algorithm aligns the promoter region, about 20 nucleotides upstream its "-35" box and the transcribed region up to the start codon (the part of the alignment is given in Fig. 1) and chooses the putative boxes taking into account the distance between them (typically 17-18 nucleotides) and their affinity on the species tree (closer species have more similar sequences). The algorithms are described in detail in [6,7].

5) Results. Why the authors insist to strengthen differences between plastid REP-promoter of psaA gene and bacterial $\sigma-70$ promoters?

Response: The psaA leader regions have a reliable long alignment, which accents the fact that this promoter considerably differs from the bacterial sigma-70 consensus.

\section{Reviewer's report 3}

Yuri I. Wolf, National Center for Biotechnology Information (nominated by Purificación López-García, Université Paris-Sud)

The authors report the virtual lack of conservation of Plastid-Encoded Polymerase promoters among the various lineages of plants. The finding is quite noteworthy and would be of interest to those who study the evolution of regulatory elements and plastid genomes.

1) p. 2. "Plastid genes and their promoters are believed to be evolutionarily conserved across large taxonomic lineages". This is a strong statement that requires at least a couple of references, indicated who, when and in what form expressed these beliefs.
Response: In [2, 9.7c] (this reference is added) the authors state that "The structure of chloroplast... genes is widely conserved across lineages. Their evolutionary rate is much lower than that of nuclear genes." This seems to be a common knowledge from textbooks (references can be added if necessary). Our logic was first straight: highly conserved genes cannot have low conserved promoters. But out results show the opposite. The phrase "and their promoters" is now removed.

2) p.3 and throughout. "The term "widely" is used to indicate...". The authors attempt to clarify the usage of the term "widely", but actually just substitute it by no less vague "across high-level taxonomic divisions". I suggest to specify the "high-level taxonomic divisions" used in the definition of "widely" and avoid the italicized usage of this term further in the text.

Response: An alignment was called "widely conserved" when included the Magnoliophyta and at least two representatives (at least one must not be a vascular plant) from Cycadophyta, Coniferophyta, Gnetophyta, Moniliformopses, Lycopodiophyta, Marchantiophyta, Bryophyta, Charophyceae, Coleochaetophyceae, Zygnemophyceae, Mesostigmatophyceae, Chlorarachniophyceae or Glaucocystophyceae. Each high lineage from Fig. 1 is represented by few species because other species can usually be unambiguously aligned. These lineages are unbalanced in terms of molecular taxon sampling and are here represented by similar numbers of species. The term "widely conserved" will hopefully be given a more precise definition in the future.

3) pp. 4-6. The gene-specific section of the Results reads like a verbal narration of the content of the Table 1. It is not clear why the authors need such a detailed listing of facts that don't seem to lead to any particular conclusions. I would recommend considering the possibility of removing this part from Results altogether, joining Results and Discussion and use the extra available space to somewhat expand the Methods section.

Response: The "Results" do not just state the fact of the widely conserved promoter and its distance from the gene (which is indeed evident from Table 1) but also comparisons of the orthologous gene promoters supported by the alignment analyses and interpretations of published data. The authors believe this section should be kept at least structurally. It might be technically merged with the Discussion but its contents should remain. Discussion elements in the Results are directly related to the details described. If the note is to be reduced, we argue for moving Fig. 2 (and, if needed, Table 1) into the supplementary data.

4) Promoter blocks for different genes seem to be aligned, but all shown sequences have different lengths. This leads to a seemingly paradoxical result - the magenta mark for the experimentally identified transcription initi- 
ation site in $p s b B$ of Spinacia oleracea highlights an empty space.

Response: The Figure 1 shows a good alignment, which length cannot be amended. If the $p s b B$ alignment is appended some columns to the right, its quality will decrease. In magenta is now a character existing in this position in spinach an experimentally proved to be at the transcription start.

\section{Additional material}

Additional file 1 The list of plastomes examined for conserved PEP

promoters. The data were extracted from GenBank, NCBI.

\section{Competing interests}

The authors declare that they have no competing interests.

\section{Authors' contributions}

VAL and AVS performed the analyses, interpreted the results and developed the algorithms. LIR programmed the algorithms and ran computations. All authors contributed equally to preparing the manuscript.

\section{Acknowledgements}

The authors are grateful to Leonid Rusin for valuable discussion and help in manuscript preparation. This study was partly supported by the International Science \& Technology Center (project no. 3807) and the Federal Agency for Education (grant no. P2370).

\section{Author Details}

Institute for Information Transmission Problems of the Russian Academy of Sciences, 19, Bolshoy Karetny per., Moscow, 127994, Russia

Received: 20 April 2010 Accepted: 10 May 2010

Published: 10 May 2010

\section{References}

1. Singer M, Berg P: Genes and Genomes Blackwell Scientific Publications Ltd. Oxford; 1991.

2. Collado-Vides J, Salgado H, Morett E, Gama-Castro S, Jiménez-Jacinto V Martínez-Flores I, Medina-Rivera A, Muñiz-Rascado L, Peralta-Gil M, Santos-Zavaleta A: Bioinformatics resources for the study of gene regulation in bacteria. J Bacterio/ 2009, 191(1):23-31.

3. Swiatecka-Hagenbruch M, Liere K, Borner T: High diversity of plastidial promoters in Arabidopsis thaliana. Mol Genet Genomics 2007, 277:725-734.

4. Hoffer PH, Christopher DA: Structure and blue-light-responsive transcription of a chloroplast $p s b D$ promoter from Arabidopsis thaliana. Plant Physiol 1997, 115:213-222.

5. Seliverstov AV, Lysenko EA, Lyubetsky VA: Rapid evolution of promoters for the plastome gene $n d h F$ in flowering plants. Russian Journal of Plant Physiology 2009, 56(6):837-844.

6. Finding of multi-box regulatory signal in the set of unaligned sequences [http://lab6.iitp.ru/en/twobox/]

7. A tree-based method of sequence alignment [http://lab6.iitp.ru/en/ treeal/]

8. Favorov AV, Gelfand MS, Gerasimova AV, Ravcheev DA, Mironov AA, Makeev VYu: A Gibbs sampler for identification of symmetrically structured, spaced DNA motifs with improved estimation of the signal length. Bioinformatics 2005, 21(10):2240-5.

9. Danilova LV, Gorbunov Klu, Gelfand MS, Liubetskii (Lyubetsky) VA: Algorithms for isolating regulatory signals in DNA sequences. Mol Biol (Mosk) 2001, 35(6):987-95. [Article in Russian].

10. Danilova LV, Lyubetsky VA, Gelfand MS: An algorithm for identification of regulatory signals in unaligned DNA sequences, its testing and parallel implementation. In Silico Biol 2003, 3(1-2):33-47

11. Rubanov L, Seliverstov A, Lyubetsky V: Multiple alignment based on species tree. Abstracts of the Sixth International Conference on
Bioinformatics of Genome Regulation and Structure, BGRS'2008: 22-28 June 2008; Novosibirsk 2008:212.

12. Homann A, Link G: DNA-binding and transcription characteristics of three cloned sigma factors from mustard (Sinapis alba L.) suggest overlapping and distinct roles in plastid gene expression. Eur J Biochem 2003, 270(6):1288-300.

13. Gruissem W, Zurawski G: Analysis of promoter regions for the spinach chloroplast $r b c L, a t p B$ and $p s b A$ genes. The EMBO Journal 1985, 4(13A):3375-3383.

14. Crooks GE, Hon G, Chandonia JM, Brenner SE: WebLogo: A sequence logo generator. Genome Research 2004, 14:1188-1190.

15. Westhoff $P$, Herrmann RG: Complex RNA maturation in chloroplasts. The psbB operon from spinach. Eur J Biochem 1988, 171:551-564.

16. Fey V, Wagner R, Braütigam K, Wirtz M, Hell R, Dietzmann A, Leister D, Oelmüller R, Pfannschmidt T: Retrograde plastid redox signals in the expression of nuclear genes for chloroplast proteins of Arabidopsis thaliana. The Journal of Biological Chemistry 2005, 280:5318-5328. Additions and Corrections, ibid, 280(17): 17572

17. Nagashima A, Hanaoka M, Motohashi R, Seki M, Shinozaki K, Kanamaru K, Takahashi H, Tanaka K: DNA microarray analysis of plastid gene expression in Arabidopsis mutant deficient in a plastid transcription factor sigma, SIG2. Biosci Biotechnol Biochem 2004, 68(3):694-704.

18. Rogers MB, Gilson PR, Su V, McFadden GI, Keeling PJ: The complete chloroplast genome of the chlorarachniophyte Bigelowiella natans: evidence for independent origins of Chlorarachniophyte and Euglenid secondary endosymbionts. Mol Biol Evol 2007, 24(1):54-62.

19. Lemieux C, Otis C, Turmel M: A clade uniting the green algae Mesostigma viride and Chlorokybus atmophyticus represents the deepest branch of the Streptophyta in chloroplast genome-based phylogenies. BMC Biology 2007, 5(2):1-17.

20. Li S, Nosenko T, Hackett JD, Bhattacharya D: Phylogenomic analysis identifies red algal genes of endosymbiotic origin in the Chromalveolates. Mol Biol Evol 2006, 23(3):663-674.

21. Lysenko EA: Plant sigma factors and their role in plastid transcription. Plant Cell Rep 2007, 26:845-859.

22. Swiatecka-Hagenbruch M, Emanuel C, Hedtke B, Liere K, Borner T: Impaired function of the phage-type RNA polymerase RpoTp in transcription of chloroplast genes is compensated by a second phagetype RNA polymerase. Nucleic Acids Research 2008, 36(3):785-792.

23. Wickett NJ, Zhang Y, Hansen SK, Roper JM, Kuehl JV, Plock SA, Wolf PG, Depamphilis CW, Boore JL, Goffinet B: Functional Gene Losses Occur with Minimal Size Reduction in the Plastid Genome of the Parasitic Liverwort Aneura mirabilis. Mol Biol Evol 2008, 25(2):393-401.

24. Seliverstov AV, Lyubetsky VA: Translation regulation of intron containing genes in chloroplasts. Journal of Bioinformatics and Computational Biology 2006, 4(4):783-793

doi: 10.1186/1745-6150-5-34

Cite this article as: Lyubetsky et al., Lack of conservation of bacterial type promoters in plastids of Streptophyta Biology Direct 2010, 5:34

\section{Submit your next manuscript to BioMed Centra} and take full advantage of:

- Convenient online submission

- Thorough peer review

- No space constraints or color figure charges

- Immediate publication on acceptance

- Inclusion in PubMed, CAS, Scopus and Google Scholar

- Research which is freely available for redistribution 